\title{
Color terms and color concepts
}

\author{
Jules Davidoff * \\ Centre for Cognition, Computation, and Culture, Goldsmiths, University of London, London SE14 6NW, UK
}

Received 23 February 2006

Available online 19 April 2006

\begin{abstract}
In their lead articles, both Kowalski and Zimiles (2006) and O'Hanlon and Roberson (2006) declare a general relation between color term knowledge and the ability to conceptually represent color. Kowalski and Zimiles, in particular, argue for a priority for the conceptual representation in color term acquisition. The complexities of the interaction are taken up in the current commentary, especially with regard to the neuropsychological evidence. Data from aphasic patients also argue for a priority for abstract thought, but nevertheless it may still be that the use of color terms is the only way in which to form color categories even if both linguistic and attentional factors play an important role.
\end{abstract}

(C) 2006 Elsevier Inc. All rights reserved.

Keywords: Color concepts; Color terms; Neuropsychology

The issue addressed in the lead articles by both Kowalski and Zimiles (2006) and O'Hanlon and Roberson (2006), but in particular by Kowalski and Zimiles, is the developmental relation between color terms and color concepts. The issue has been of considerable interest to Davidoff, Roberson, and their colleagues. In their work (e.g., Davidoff, Davies, \& Roberson, 1999; Roberson \& Davidoff, 2000; Roberson, Davidoff, Davies, \& Shapiro, 2005), possession of a color concept goes hand-in-hand with the demonstration of within-category similarity and between-category dissimilarity, that is, categorical perception. Davidoff and colleagues (1999) found that memory and perceived (category) similarity were simply predicted by the color terms in a speaker's language.

\footnotetext{
* Fax: +44 2070785145.

E-mail address: j.davidoff@gold.ac.uk.
} 
Their neo-Whorfian stance (Davidoff, 2001) derived from the cases where categorical perception is or is not found and has provoked some controversy (Franklin, Clifford, Williamson, \& Davies, 2005; Pilling, Wiggett, Özgen, \& Davies, 2003).

Pilling and colleagues (2003) disputed the apparent contention in Roberson and Davidoff (2000) that categorical perception for color derives from matching the stimulus to a color label. However, simple verbal labeling as an explanation of categorical perception is not really what those authors claimed. The current commentary gives a needed opportunity for clarification of their position. On the one hand, there is reason to believe from their data (Roberson et al., 2005) that color labels are much involved. To borrow a phrase from visual neurophysiology, Roberson and colleagues (2005) showed an "exquisite tuning" between color labels and categorical perception within a narrow range of categories. On the other hand, their other data would imply that color labels per se were not important for production of categorical perception. For example, one of Roberson and Davidoff's (2000) experiments showed that interference from noncolor words was equally effective as that from color words in removing categorical perception. Furthermore, O'Hanlon and Roberson (2006) give several examples, particularly from developmental studies, where there is no clear relation between performance on color tasks and the speaker's basic color knowledge. A reflection on these conflicting directions is given here from a consideration of what might at first seem to be a quite different field of research.

A similar conflict about the role of color labels was shown in a patient, LEW, who could neither produce nor comprehend color terms, and although he was unable to understand color category similarity, he nevertheless showed normal categorical perception (Roberson, Davidoff, \& Braisby, 1999). Thus, evidence from neuropsychology may be critical to understanding the relation between color terms and abstract color concepts. The important evidence comes from studies of patients with language impairment (aphasia) in categorization tasks. These tasks have been used by many neuropsychologists because an inability to categorize was considered crucial to the debate concerning the relations between impaired language and thought. During the early days of modern neuropsychological research, Wernicke considered aphasia to be a lexical impairment (see Davidoff \& Roberson, 2004). In contrast, Hughlings Jackson (cited in Zangwill, 1964, p. 261) declared that aphasics, by having lost language, were "lame in thinking." The argument was considered one of the most crucial in neuropsychology and surfaced many times during the subsequent 100 years. Indeed, Goldstein (1948) considered that a loss of abstract processing was the core deficit in aphasia.

As Goldstein (1948) noted, there are two main types of categorization task. One type can be "solved" simply by episodic knowledge and what he called a concrete attitude, and the other type required an abstract attitude. According to Goldstein, after certain types of aphasic damage, the patient maintained only a concrete attitude. For color tasks, the consequence is that judgments are based on perceptual similarity. For other tasks, the patient's judgments are based on what are now termed thematic associations (Markman \& Huchison, 1984) or on personal associations. For example, Goldstein (1948) described a patient who kept both a hammer and a saucepan in the kitchen and therefore erroneously placed them together in a categorization task. Another example, where the use of episodic (visual) associations arrives at correct categorization, is from LEW (Roberson et al., 1999), who was able to sort animals into British versus foreign by virtue of whether he had seen them in a zoo. 
LEW was completely unable to sort colors (Roberson et al., 1999). A further examination of the patient (Davidoff \& Roberson, 2004) showed clearly that the difficulty in sorting colors was part of a general difficulty in taxonomic classification. His difficulty was not explained by a general intellectual deficit. LEW was also asked to do analogical reasoning tasks of the type used by Gentner (see Kowalski \& Zimiles, 2006). Although his level of performance on analogical reasoning was only that of a 4- or 5-year-old, it far surpassed his ability to follow rules of perceptual classification. It was therefore argued that taxonomic classifications cannot be driven by the development of analogical reasoning. However, LEW behaved like a 3-year-old in a task that directly contrasted thematic and taxonomic classification (Markman \& Huchison, 1984). LEW showed a preference for thematic classification. In fact, there was no evidence of any substantial ability to make taxonomic color classifications despite evidence for good preservation of the associated object-color knowledge (e.g., red went with strawberry). The reason is that tasks involving colors, as opposed to object-colors, allow only a limited number of procedures to achieve correct categorization. For example, artifacts and animals, because of their multiple associations and properties, allow multiple routes for categorization. Indeed, allocation of objects to categories is always context dependent; there are an infinite number of ways in which they may be divided into categories. Colors are not like objects because there is not the variety of episodic knowledge to use in color categorization tasks. Thus, the failure of the patients is more extreme for perceptual categories because there are no associations, such as are seen in a zoo, that can be used in the task of color categorization.

These important differences between objects and colors with respect to categorization are laid down in the model of color processing proposed by Davidoff (1991). That model proposed an internal color space that would organize together perceptual hues defined as similar. Thus, access to the color space was required to produce a common lexical entry to many different hues. However, the model may be incorrect in showing that color terms can be accessed only by reference to the internal color space. It must be possible to "parrot" a color term without its understanding. So, it might be better put to say that the internal color space is used only when, to use Goldstein's (1948) terms, we adopt an abstract attitude for color naming. Goldstein contended that when a person "truly 'names' an object, he has the experience of a word which 'means' this object. ... Otherwise, he experiences the word as a sound complex belonging to an object" (p. 61). Goldstein referred to the type of naming that is preserved without access to the semantic features of an object as "pseudonaming," in contrast to true naming that requires an abstract attitude.

Goldstein (1948) claimed that when pseudonaming, the names do not affect thought. The consequence is that the label given to the color patch does not influence categorization. For example, Goldstein reported a patient who placed colors in the same group despite an unwillingness to call them by the same name. When asked whether the colors in the group could not all be called red, the patient replied "No, these are red, this one is pink, this one is maroon." LEW's poor color sorting was carried out in much the same way and likewise gave away his inability to employ an abstract attitude. Goldstein warned that the patient's placing of color stimuli in a line according to some similarity dimension should not lead the examiner to believe that the colors were categorized; rather, the reverse is the case. He argued that the patient's sorting behavior differed from that used in normal categorization. When asked to sort many colors, we normally make piles of colors; this is possible because the task is driven by some internal categorization mechanism, possibly 
the name. The person has no need to see the colors already classified. In contrast, the patient with only concrete abilities needs to make visual, and apparently only pairwise, comparisons. The patient's concrete sorting procedure requires that stimuli that have already been classified be in sight.

LEW's sorting of colors (and shapes) was restricted to that which can be achieved by perceptual similarity. The normal person also has the ability to sort categorically but can adopt either mode. Kay and Kempton (1984), by varying task instructions and procedures, were able to induce normal adults to make "odd one out" color judgments based either on perceptual or linguistic (categorical) differences. Recently, these two types of judgment have been again related to a language versus a perceptual attitude to processing with consequent implication for hemispheric differences (Gilbert, Regier, Kay, \& Ivry, 2006). The categorical effects were found only for left hemisphere presentations.

The neuropsychological evidence would seem to give priority for a general semiotic ability responsible for color concepts that could precede the acquisition of color terms. Therefore, as both lead articles in this issue show, children may understand about color before knowing color terms. However, things are not that simple. One needs to explain the origin of the particular color concepts. Roberson and colleagues (1999) argued that there was no other source for the origin of color categories besides labels unless the concepts had some innate basis as, for example, proposed by Franklin and colleagues (2005). One argument against an innate basis is that it would mean a considerable amount of unlearning for speakers of most of the world's languages where categories (e.g., blue, green) do not exist in the adult's language.

Recently, Fagot, Goldstein, Davidoff, and Pickering (in press) examined the question of whether color categories are innate from a different approach by observing the similarity matching of baboons and humans. They used as standards a typical green and a typical blue. The interest was in the match of the intermediary colors. None of the baboons, despite having color discrimination as good as that of humans, showed any inclination to match any but very close colors to the standard. Humans showed quite different performance, with a sharp division of the intermediary colors at the appropriate boundary between green and blue. At the very least, Fagot and colleagues showed that the possession of a trichromatic visual system like that of humans does not by itself produce color categories. So, one is drawn back to the argument that, at the very least, labels generate the range of each color category.

In light of this work, what are the conclusions that we ought to draw from the Kowalski and Zimiles article and the O'Hanlon and Roberson article? Kowalski and Zimiles show that 3-year-olds have an idea about color and can extract color as a feature and use it in an identity match. Specific color labels do not seem to map neatly onto those specific color concepts, but overall there is a good relation between the two abilities. Importantly, there is a priority for the abstract representation. The current commentary does not disagree with these conclusions, but it cautions that the relation between concepts and terms may be more profound than is shown in their study. A similar caution may be given to the studies of O'Hanlon and Roberson in which they assign the relative importance of linguistic or attentional factors to the acquisition of new color labels. Their tasks, like those of Kowalski and Zimiles, do not allow us to know whether children really understand about redness, blueness, beigeness, and the like. To be sure about that level of abstraction, we need to examine the range of the match. So, if the interest is to examine the question of whether labels are necessarily required for color category instantiation, a more demanding 
task-one that uses similarity rather than identity matching-could show a precise connection.

\section{Acknowledgment}

The author is supported by an EU Grant (12984), Stages in the Evolution and Development of Sign Use (SEDSU), and by a Leverhulme major research Grant (F/07 605/D).

\section{References}

Davidoff, J. (1991). Cognition through color. Cambridge, MA: MIT Press.

Davidoff, J. (2001). Language and perceptual categories. Trends in Cognitive Science, 5, 382-387.

Davidoff, J., Davies, I., \& Roberson, D. (1999). Colour categories of a stone-age tribe. Nature, 398, 203-204.

Davidoff, J., \& Roberson, D. (2004). Preserved thematic and impaired taxonomic categorisation: A case study. Language and Cognitive Processes, 19, 137-174.

Fagot, J., Goldstein, J., Davidoff, J., \& Pickering, A. (in press). Cross-species differences in color categorization. Psychonomic Bulletin and Review.

Franklin, A., Clifford, A., Williamson, E., \& Davies, I. R. L. (2005). Color term knowledge does not affect categorical perception in toddlers. Journal of Experimental Child Psychology, 90, 114-141.

Gilbert, A. L., Regier, T., Kay, P., \& Ivry, R. B. (2006). Whorf hypothesis is supported in the right visual field but not the left. Proceedings of the National Academy of Sciences, 103, 489-494.

Goldstein, K. (1948). Language and language disturbances. New York: Grune \& Stratton.

Kay, P., \& Kempton, W. (1984). What is the Sapir-Whorf hypothesis? American Anthropologist, 86, 65-78.

Kowalski, K., \& Zimiles, H. (2006). The relation between children's conceptual functioning with color and color term acquisition. Journal of Experimental Child Psychology, 94, 301-321.

Markman, E., \& Huchison, J. (1984). Children's sensitivity to constraints on word meaning: Taxonomic vs. thematic relations. Cognitive Psychology, 16, 1-27.

O'Hanlon, C., \& Roberson, D. (2006). Learning in context: Linguistic and attentional constraints on children's color term learning. Journal of Experimental Child Psychology, 94, 275-300.

Pilling, M., Wiggett, A., Özgen, E., \& Davies, I. R. L. (2003). Is color "categorical perception” really perceptual? Memory \& Cognition, 31, 538-551.

Roberson, D., \& Davidoff, J. (2000). The categorical perception of colors and facial expressions: The effect of verbal interference. Memory \& Cognition, 28, 977-986.

Roberson, D., Davidoff, J., \& Braisby, N. (1999). Similarity and categorisation: Neuropsychological evidence for a dissociation in explicit categorisation tasks. Cognition, 71, 1-42.

Roberson, D., Davidoff, J., Davies, I. R., \& Shapiro, L. R. (2005). Color categories: Evidence for the cultural relativity hypothesis. Cognitive Psychology, 50, 378-411.

Zangwill, O. L. (1964). Intelligence in aphasia. In A. V. S. de Reuck \& M. O'Connor (Eds.), Disorders of language (pp. 261-284). London: J. \& A. Churchill. 